\title{
2. Artnote: Data as Performance
}

\author{
CHRIS RABIDEAU AND KAREN ROLAND
}

\begin{abstract}
This description of the Artnote is included as a means with which to share with the reader our perspectives concerning the development of an innovative approach to presenting data, specifically, the research findings of the Tikkun youth researchers through an arts-based ethnographic (personally immersive and reflexive) practice - a method of presenting research data as performance. The Artnote, rather than a keynote address, was presented at the Tikkun Youth Symposium to facilitate the inclusive participation of youth researchers from all five of the Tikkun research sites. In this chapter the following aspects of the Artnote will be discussed: the vision for creating the Artnote to celebrate the voices of youth; data as performance as the means used to engage youth, researchers, and the audience in meaningful discussion, and further, to develop an experiential understanding of the data presented; and the use of the Artnote as a novel approach for mobilizing knowledge and thereby fostering the voice of youth leadership in social justice research and activism.

Key Words/Phrases: vision for the Artnote; data as performance; fostering the voice of youth leadership in social justice research and activism, mobilizing knowledge
\end{abstract}

This description of the Artnote is included as a means with which to share with the reader our perspectives concerning the development of an innovative approach to presenting data, specifically, the research findings of the Tikkun youth researchers through an arts-based ethnographic (personally immersive and reflexive) practice - a method of presenting research data as performance. By "performance," we mean that rather than a keynote address to present the research findings from each of the Tikkun research sites, the Artnote was presented at the Tikkun Youth Symposium 
to facilitate the inclusive participation of youth researchers from all five of the Tikkun research sites, along with the audience. The Artnote was created as an interactive and experiential performance which attempted to create "a transformative 'liminal space' for the audience" (Bagley, 2008, p. 67). In other words, the objective of the Artnote was not only to disseminate research data, but also to immerse the youth researchers and the audience in an experiential learning event. The goal was to share stories and insights through this creative process, and to engage with and increase our knowledge and view of the world from a global perspective.

In this chapter, the authors will present their perspectives of the following: the project team vision for creating the Artnote as a means of celebrating the voices of youth; presenting data as performance to engage youth, researchers, and the audience in meaningful discussion, and further, to develop an experiential understanding of the data presented; and the use of the Artnote as a novel means with which to mobilize knowledge and thereby foster the voice of youth leadership in social justice research and activism. As members of the Tikkun Youth Project, we developed this chapter about the Artnote through a collaborative writing process which included a discussion of storytelling and a focus on the arts as media for expression and knowledge mobilization. Knowledge mobilization is an essential aspect of research. In our work, knowledge mobilization was viewed not only for the purpose of disseminating research findings to add to the body of knowledge about social justice and activism, but also, through the Artnote presentation, a means with which to provide the added element of access to the research findings through an experiential lens - data as performance. To ensure that we were able to convey a nuanced understanding of the Artnote and its development, implementation, and ability to engage and empower youth, some sections of this chapter offer a narrative that was collaboratively developed.

\section{The Artnote Vision}

Have you ever attended a lecture or research symposium and felt that while intellectually you may have understood what was being shared, you did not feel a personal connection to the research findings presented or the experiences of the researchers? Did you walk away without having 
experienced a personal understanding of the research and the findings? Did the research data and the findings shared by the researchers affect your world view? These were central concerns and a focus of the members of the Tikkun Youth Project - the youth researchers not only wanted to disseminate their research, but also wanted to act as agents of change and to present their research data in a manner that further encompassed their identity as global social justice activists and advocates to promote social change. Therefore, it was essential that the Artnote be developed as a collaborative and educational arts-based initiative based on the research data from each of the Tikkun project research sites; this multi-epistemic data included ideas, stories, poems, songs, and the personal experiences and narratives of youth researchers. Built upon the foundation of the Tikkun philosophy of healing and reconciliation, the Artnote was collectively created to reflect the social, educational, and environmental factors that were concerning from a youth perspective about the world, and how we as citizens of the world can work together to begin to heal and to answer the call of social justice. As an ethnographic data performance strategy, the Tikkun youth researchers ensured that the Artnote embraced the power of the story to illustrate truth, lived experience, empathy, and understanding. As Bell (2010) asserted, "Stories are one of the most powerful and personal ways that we learn about the world.... stories operate on both individual and collective levels, they can bridge the sociological, abstract with the psychological, personal contours of daily experience" (p. 16). In the remainder of this chapter, the term "data" will be used to describe the research findings shared and experienced by the Tikkun researchers as described above.

Following Dewey's (1938) assertion, "the principle that development of experience comes through interaction means that education is essentially a social process" (p. 38); the process used to develop the Artnote was a shared collaborative and social experience during which the youth researchers from each of the Tikkun project sites were able to share their stories, insights, and reflections. In doing this, the youth researchers shared the data from their research in a creative manner. The Artnote participants, as co-creators in this process, were given the opportunity to identify the data that were used and then to choose the focus of their narratives and artistic expression to be included in the Artnote performance. This was done through the following epistemologically reflexive processes: reflections of the self in the world, reflections of social duty and responsibility, and reflections on 
the world (worldview). Through these reflective processes, and by using creative expression, the participants were able to identify specifically what art form they wanted to use to present their research data. The options included drama, dance, visual arts, poetry, music, video, or any other art form they wished. The element of choice was critical for this creative process to respect the dignity of the youth researchers, ensuring their comfort, and most importantly, the authentic representation of the research data they were sharing - data as performance.

\section{Creating the Artnote}

The process of creating the Artnote began in the spring of 2016 when Chris Rabideau, an award-winning social justice, community-engaged artist, was invited to join the Tikkun Youth Project as Artistic Director for the April 2017 Tikkun Beyond Borders: Connecting Youth Voices, Leading Change Symposium. As an artist and educator, Chris was an ideal candidate given his extensive background working with youth locally to create theatre-based performances and projects that address social justice issues; he has worked with community not-for-profit organizations, school boards and community groups. His thoughtful and intentional respect for youth and their "voices" was demonstrated by his ability to be flexible and fluid within the artistic process. 1

We believe that the youth researchers' vision of the Artnote was that of a "call to action." As the authors of this chapter, we have collaborated to develop the story behind the Artnote - its inception, creative process of development, and outcomes. Our discussion in this chapter of the Artnote includes a narrative we developed collaboratively. We have done this to ensure that the meaning and nuances of the creative process are described accurately. In this way we have worked together to share these perspectives, as noted below:

When meeting with the Tikkun project team to discuss the concept of a keynote presentation for the 2017 Symposium, we were intrigued by the prospect of youth from different communities around the world gathering together to share their research and thoughts about "repairing our world" through youth activism and advocacy. As we began to share ideas, it was evident that at the 
core, the Tikkun Symposium objective was to celebrate the culture, identity, and individuality of the youth researchers from each of the five project sites. The Artnote would essentially be an opportunity to use the arts to explain, and most importantly, experience educational research.

The Arts are a powerful medium for expression. They provide an authentic communication process that can create an educational and empowering experience with the potential to affect positive social change in our communities and institutions. Arts education, as a pedagogical approach, provides individuals and groups with an educational experience by which they may empathically step inside the shoes of the "other." For the youth researchers we saw this process as the means by which to develop their self-confidence, leadership, and communication skills. The Arts have the power to illuminate social issues such as discrimination, stereotyping, and violence, while developing awareness and empathy in our communities and our classrooms. As social justice artists and advocates, we knew that this was the way to provide the youth researchers with the opportunity to share their stories, and to bring not only a piece of home with them, but also to showcase their talents through the Arts. An example of this was illustrated through the performance and video created by the Thunder Bay youth researchers to share their research data about the experiences of First Nations youth in navigating curricular challenges, having to travel in some cases up to a thousand miles or more to attend school, and therefore having to be absent from home for many months at time - all in the pursuit of education in Ontario. Their research findings were articulated through the performance of their research data during the Artnote (Figure 1). 


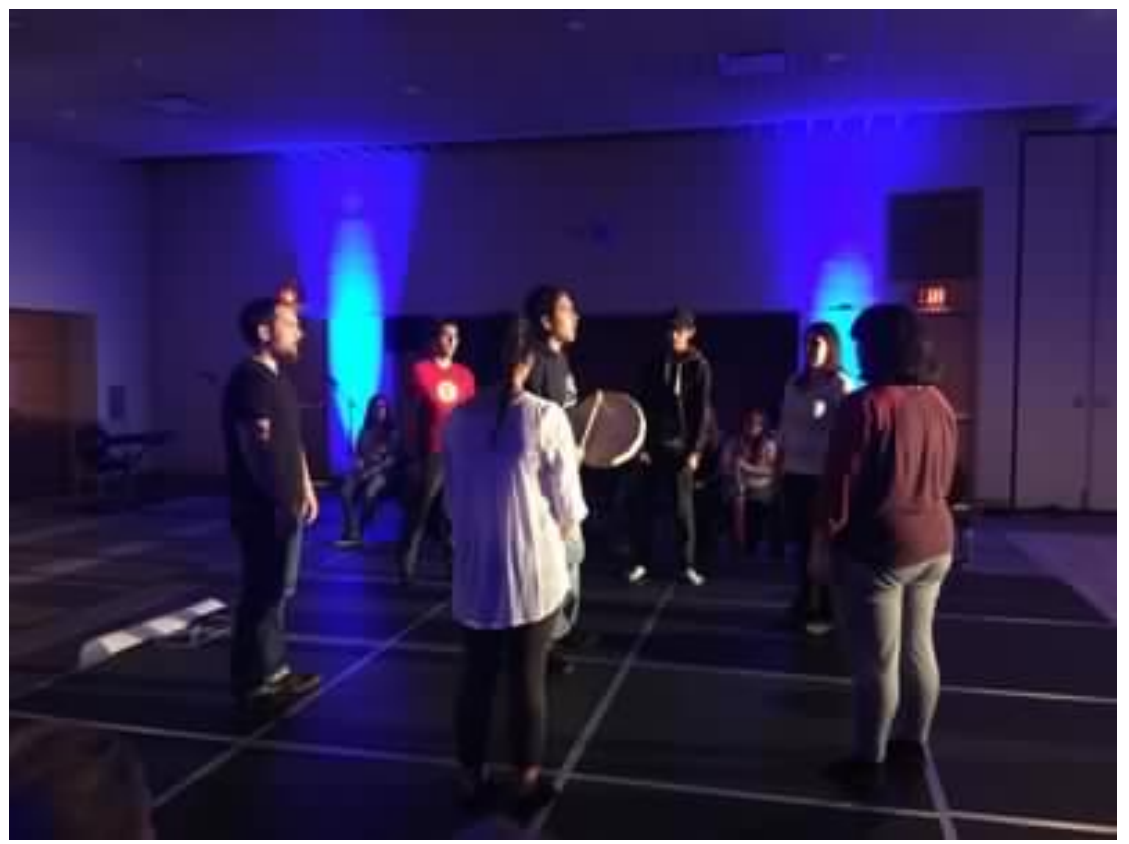

Figure 1. Thunder Bay researchers' Artnote performance.

\section{Celebrating the Voices of Youth}

An essential aspect of our collaboration to create the Artnote began by contacting the Tikkun researchers at each of the project sites (South Africa, Kosovo, Thunder Bay, Toronto, and Windsor) prior to their arrival at the University of Windsor; the initial steps of this process are noted:

As we started to plan, we realized what a challenging undertaking the Artnote was going to be. This was exciting and yet concerning too: Could we effectively translate this excitement into action? Several other questions also came to mind: How much time would we have to put this collaborative presentation of research data together as performance? How would we begin? Where would be the best venue to hold the Artnote, and what would be the needs in this creative space to ensure an interactive audience experience? Would the youth researchers involved even like the idea of an Artnote? 
The goal was to provide youth researchers with a unique and creative process through which to mobilize knowledge about social justice issues and thereby ensure that their "voices" about how they, as youth, have been answering the call of social justice in their communities. The process unfolded organically by allowing the voices of the youth to guide us. In gathering background information, we learned that many of the youth researchers had been working in their home communities on a diverse range of social justice projects and that in some cases, they had brought with them fully formed arts-based performance concepts such as songs, poems, and/ or dance. However, the central focus of all of their work was to make change and to promote the voice of youth as change agents. At each of the Tikkun project sites, this focus was clearly articulated, as was the need to address social justice as a call to action led by youth. In addition to the Windsor site's call to action for economic justice through their Fight for \$15 \& Fairness project, during our initial meeting at the University of Windsor campus, the youth researchers from the other Tikkun project sites also identified the following calls to action: "Thunder Bay site - Clean water for all; South African site - One school, one library, one librarian; Toronto site - Tuition way too high, you need to cut it; and Kosovo site - No walls, more bridges!" All of the Tikkun projects collectively were a global call to action - youth leading social change!

The development of the Artnote was similar in many respects to Bell's (2010) Storytelling Project Model in that there was a concerted effort to create a "community of diverse members in which stories" (p. 20) could be shared. Bell's model consists of four connected story types: stock stories, concealed stories, counter-stories, and stories of resistance. The "stock stories" are those perpetuated and told by mainstream society. They contrast with the "concealed stories" which Bell identified as those told and kept secret by marginalized groups. These concealed stories effectively "portray the strengths and capacities within marginalized communities" (p. 23). The national pride and cultural heritage expressed by the Kosovo youth researchers may be considered an example of concealed stories. Counterstories also consciously seek to disrupt "stock stories" through the storytelling process. Bell's storytelling model also incorporates stories of "resistance" that articulate how marginalized groups defy and challenge the discrimination perpetuated by societal "stock" stories. The South African youth researchers presented research data that illustrated these stories of resistance - they chose to use their voices in song, poetry, action, and 
improvisation to recreate life at home and to represent the data collected as articulated in the song performed as part of the Artnote script: "My Mother was a kitchen girl, My Father was a garden boy; that's why I'm an activist, that's why I'm an activist!"

Finally, in her work, Bell (2010) suggests that the Storytelling Project Model fosters the creation of "emerging/transforming stories" - new stories that not only challenge the stock stories, but also build upon the messages of truth and activism to "interrupt the status quo and energize change" (p. 25). Storytelling may require some messiness and move people outside of their comfort zones; however, this messiness and tension are necessary aspects of the process, and this was definitely experienced during the creation of the Artnote performance. Some of the impressions concerning guiding the youth researchers to develop their stories through an arts-based process including the following:

The creation of the Artnote presentation was not an easy task. Initially, each of the project sites were contacted, but there were many unanticipated communication difficulties such as access to the Internet and working with different time zones that proved to be quite challenging at times. Once each site was contacted, we began by discussing with the youth researchers the idea of presenting their research data at the Symposium using an Artnote presentation. Initially, we thought that they would love the idea. However, as we discussed the concept further, they began to realize the scope of what was being asked of them. As the objective of using data as performance started to set in, most groups began to respond with concern and fears: So, what is it exactly that you want us to do? What do you mean by "artistic"? Am I going to be on stage and required to act? I don't understand what you mean. This stage in the Artnote development began to feel like a very disorganized and chaotic classroom where the lesson is not going well! However, everyone was clearly engaged!

At this stage we took a step back with the realization that many of the youth researchers may never have had an experience within the Arts in terms of an arts-based experiential performance. The unstructured and creative aspect of using the arts as a medium to present research data felt foreign and difficult. The participants struggled with conceptualizing the end product 
- this was especially difficult given that the Artistic Director did not know what it would "look like" either. The uncertainty was unnerving to some. We discussed that this was part of the creative process and that experimentation is an essential aspect of the arts and the creative process. However, we were cognizant that living in the unknown can be difficult for people particularly as we live in a society of yes and no, black and white, where boundaries and rules guide our thinking. We have found that the arts can provide endless possibilities; Jenna, a youth from the Toronto site, eloquently captures this spirit of possibilities in her poem included in the Artnote script:

What is a rainbow but an optical illusion? A series of 42 degree angle refractions of light,

Creating the appearance of colours, One falling naturally on top of the other

It is said that no one experiences the exact same rainbow Rather, it is dependent on each specific angle and perspective, Each unique reflection

It is an intersection of light and water and oneself Which results in a both beautiful and orderly

deception Placing one above the other, but this vision a regression

The community is trapped within a fallacy of hierarchies, Where levels of exclusion dismiss one's

identity when it is too far from comprehensive ability

Where gay is okay, but only when it matches the correct shade, And where you can use the

washroom beside me, but only if you stay at least two feet away

Trapped in a thought wave that one's oppression dismisses the opportunity to be the oppressor

And that love is love is love, and the rights to love are fought and gotten But when this love

does not speak the same language, it is quickly forgotten

What is a rainbow but an optical illusion? A system of hierarchy which reflects a method of

exclusion This is a challenge to claim that all love is truly love, and all rights are everyone's

rights, to question which colour and voice in this hierarchy is prioritized.

No matter who is the apple of your eye, or what is between one's 
thighs,

A challenge to make sure each colour is visible, no matter how one's queerness is identified.

Therefore, to engage in possibilities to create the Artnote, it was necessary to find that empathetic place within ourselves so that we could understand each other. In responding to the youth researchers' concerns and fears of the creative process, the goal was to break down walls, not start putting them up. We had to acknowledge that the youth researchers were looking for structure, but the fact was that they were the architects of this structure - the Artnote was their creation. We explained to the Tikkun youth researchers that we wanted them to guide us as they were the authors of the field experience and research data. Similar to Bell's (2010) Storytelling Model, this creative process led to further but nonetheless necessary confusion, which ultimately, through consistent guidance and support, resulted in the creation of an Artnote presentation.

Essential to the success of this collaborative creative process was the consistent support and mentorship provided by the Windsor youth researchers as the host project site. Their efforts proved key to our collective achievement of an Artnote presentation! Prior to all the Tikkun sites joining us at the Symposium in Windsor, the Artistic Director had the opportunity to work closely with the Windsor project participants so that they could embrace the role of host site. We wanted to ensure that the artistic pieces created by the Windsor group, based on their Fight for \$15 \& Fairness project for economic justice, would ultimately unite the project as a whole, as well as provide a loose structure for the preliminary design of the Artnote. We did not have much access to what the other Tikkun project groups were doing prior to their arrival at the University of Windsor, Faculty of Education, and we wanted to make sure the Windsor group gave us a broth in which to create our artistic soup.

Therefore, from the perspective of the Tikkun youth researchers and the Artistic Director, the Artnote proved to be an ethnographic educational strategy which reflected many of the stories as identified by Bell (2010) in her Storytelling Model, and which carefully embedded reflective practices by using the arts as the medium of research dissemination - data as performance. This was clearly demonstrated as creation of the Artnote was driven by the research data in the form of personal stories and reflections by the youth and research participants. This was a truthful and authentic 
approach to developing the performance. As Prus (1996) shared, the arts have always been a powerful and impactful medium for conveying the authentic narratives of humanity.

\section{Data as Performance}

The Artnote was comprised of two essential elements: art and note. The first focus was on the art, referring to the artistic process and practice, which showcased the development and form in which participants delivered ideas and contributions through an artistic prism. The experience of developing and performing the Artnote was supported by the literature indicating that the arts can offer a limitless world of opportunity to share insights and research findings. Through their participation, the Tikkun youth researchers were able to share their stories and develop an artistic approach to showcase their research data. The other key component of the Artnote was the opportunity that the audience was provided with to engage in the lived experiences and narratives of the youth researchers - data as performance. This other aspect, the note, was an ethnographic educational strategy that allowed all participants of the Artnote, including the audience, to have a collective experience with which to promote an empathic understanding of the data presented. The note was what was left with the audience an impression, a feeling. This became the signature, the human imprint of research data as performance: the audience reflected on the process as the performance was unfolding. Just like reading a text and having an opinion, the audience was left with having lived an experience that attempted to provide them with an empathic understanding of the research data.

The Artnote sought to disrupt, challenge, and engage the audience with the research data - not as passive receivers of information, but rather as engaged constructivists of a new reality. They were engaging in what Bell (2010) opined as a "different kind of listening" (p. 47), given that the Artnote audience was provided with the opportunity to "experience" the research data, to empathically listen to the lived-experiences shared through the data. The result of this experiential process was to challenge the audience's ways of knowing the world, and thereby underscore a developing understanding of the experience of others. Through this engagement, the participants both within the process and those who witnessed the Artnote performance were 
given the opportunity to step inside the shoes of another and be part of a transformative experience. This was a rare research opportunity: data were not only disseminated, but also shared as a collective experience. This collective experience was congruent with Dewey's (1934) assertion that, "the arts remove the veils that keep the eyes from seeing" (p. 325). Talisha, a Toronto youth researcher, communicated this "removing the veil" when she spoke about feminism in this excerpt from the Artnote performance:

I wonder,

Is your feminism my feminism?

Does your feminism fight my fight

Walk my walk

Talk my talk

Who is your feminism for?

Is it for the Muslim women who are constantly being targets for hate crimes

Is it for the black women who march on the front lines?

Is it for the indo-Caribbean women who never seem to belong

Is it for the missing and murdered indigenous women?

Do you march with BLM, no justice no peace

Do you march to the strawberry ceremony?

Have you marched for people who look like me?

So I ask who is your feminism for?

\section{Building the Show}

In discussing the stages of development for the Artnote, including the initial creation of the Artnote idea, the Tikkun project team considered: How could an Artnote be a means of presenting research data? What other aspects of the Artnote could be developed and explored? What changes were made to the initial Artnote idea as each Tikkun project site worked on their data as performance? What was the impact of the convergence of arts-based and non-arts-based learners in the process? What were the challenges? During the first contact with individual research sites, what were the challenges that no one saw coming? How were these challenges navigated? What approach was used, and did this approach work? During the symposium, how did the 
research project sites coalesce data to weave together a collective Artnote presentation - data as performance? These were questions central to the collective and collaborative process used in creating the Artnote, data as performance:

What we wanted was for each Tikkun project site to use an arts medium such as dance, drama, poetry, video, song, etc., to showcase the injustices that they witnessed or experienced in their communities via presentation of their research data. The role of the Artistic Director was to weave their stories together. Underlying this process was the understanding that the arts provides an environment in which both experiential tasks and idea-making create more experiences to form a presentation structure.

Personal narratives and storytelling can be an explorative way to guide a project. To guide the participants through a web of stories, we challenged the youth researchers to consider: How do your stories connect? What common themes are developed? What do we want to say, and how can we say it? The youth researchers were not only contributors, but also the authors of the Artnote. Initially, the youth researchers had been seeking a structured beginning, middle, and end to the Artnote, and the uncertainty of weaving their research narratives into the collective Artnote was unnerving.

As noted previously, the creation of the Artnote began through initial contacts made by the Artistic Director to the various Tikkun project site participants. However, we suggest that the creative process really began in earnest once all the youth researchers convened to form a collective body of researchers at the University of Windsor, Faculty of Education. In retrospect, many aspects of the theory of stages of small group development that were identified by Tuckman and Jensen (1977) were displayed during the development of this collective as a community: forming, storming, norming, performing, and transforming. Getting to know each other, sharing research, and sharing personal stories were all aspects essential to forming a collective identity. Sharing intellectual and physical space was part of the storming and norming aspects of collective development. Furthermore, the process of creating the Artnote gave youth the opportunity to develop as a community of researchers through the stages of performing and transforming:

When the youth arrived, we in Windsor felt a great sense of responsibility. Before we could move ahead with the project, it was 
imperative that we took the time to get to know one another. Any dramatic endeavour needs to begin with all parties understanding each other, and where the "other" is at. This meant that our first goal was to get to know the youth researchers and to reach out to them on a personal level. We did our best to carefully observe and attend to everything that was said - and to what was not said. We had to get to know each other, and in doing so, we had to learn to trust each other. In this trust-building process it is often best to start by sharing oneself - putting yourself in a personally vulnerable position to foster both trust and community-building. To facilitate this, the entire group was given the opportunity to take part in a number of trust exercises and activities as part of their orientation. These activities proved to be very helpful. Everyone needed to trust each other. That's where we had to start - earning trust.

This need to earn trust to foster the creative process was also evident during initial work with the Windsor project site - the Artnote process really began with building trust with the members of the host site. With the Windsor project site, we started to build trust by "playing" together. When we are children, we play not for sport but for fun. When someone wins, "play" becomes a game or sport. However, by playing we learn how to share, grow, and develop as individuals. Adults, it seems, have forgotten how to play. Through play and efforts to consciously build a sense of community, we began to trust each other.

With this sense of community and trust, when meeting with all the project sites, although we were aware that we had very limited time to create our collective performance, each group was encouraged to experiment and to draw upon their experiences and their research data. Following this, the youth researchers were invited to share with each other in a "show-and-tell" exercise to reveal their preliminary plans for presenting their data. This was very helpful. When these sharing presentations were completed, each group was asked questions to guide them in weaving their stories together into a collective Artnote performance. In all, the Artnote was conceived, written, and presented within a 2-day period! The narratives, which were based on the research findings (data) and stories shared, were used to build a final blueprint for the Artnote, which included staging the performance.

To effectively create the experiential aspect of our performance, the stage was designed to consist of 12 to 16 riser platforms with the cast seated with 
the audience on three sides. This was done to allow the cast to sit when they were not on stage. The performance was intended to be completely in the round, meaning that the audience would be seated in a circle arrangement around the perimeter of the stage area; seating was an essential consideration as we wanted to ensure our ability to immerse the audience experientially so that they were able to empathically understand the diverse worldview and experiences portrayed by the youth in their performances. Ultimately, given the space available and the requirements of the performance, the staging of the show was more of a "thrust stage," with the audience seated along 3 sides of the stage, allowing a back wall to be used as a screen for projected images and providing a dedicated space for musical instruments.

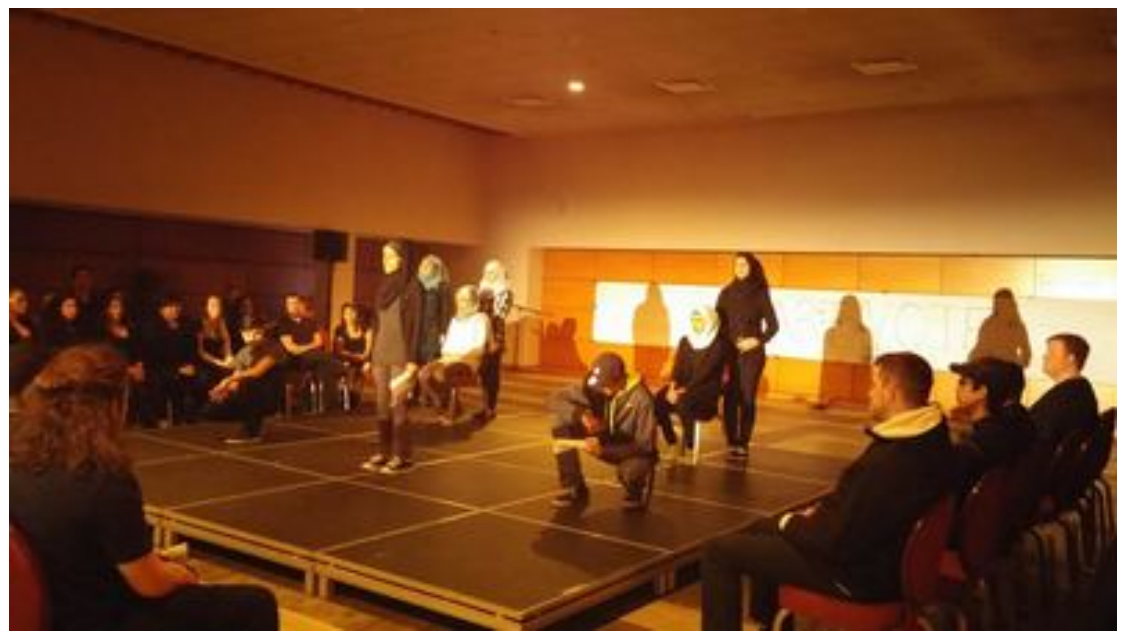

Figure 2. Creating the Artnote.

This novel approach to data as performance underscored how we as research participants may reflect through an arts-based activity, and how our reflexivity may be presented in the form of data dissemination as performance. Freire (1985) stated that the process of "realizing a theme" requires that one must reflect and acknowledge our personal and epistemological "truth" of reality, and that this notion of "truth" has a significance on the origins of the "theme" we then develop (p. 112). In other 
words, our actions - and our reflections on these actions - transform the world by which we are transformed. Through the Artnote, as collective participants, we were able to engage in reflective expressions as "reflection to self" and "reflection to our world." Some of the youth researchers were experiencing, for the first time, being involved in an arts-based performance to experientially present research data. This, in turn, may have created difficulty for some to conceptualize the meaning and feeling of the performance until the Artnote performance was fully realized and experienced. The arts are a continuum, always moving and evolving. People often look at the end product as the learned experience; as educators, we have found that for many students the final mark they receive in a course is how they determine what is learned. However, the arts continue to push us and teach us during the process, through the experience, as well as after the experience has concluded; this experiential learning process provides an opportunity for the learner to rethink their worldview, their epistemological (way of knowing) positions as well as, most importantly, allowing for greater empathy and understanding to see another's point of view. The arts, through the creative process, can stimulate deeper thought and introspection and ultimately lead us to action. The process is just as important as the final result. In many ways, the process is rather more important than the end product. The "play," the questioning, and the learning-though process are what nurture the participant's growth and development.

An example of this learning-through process during the Artnote was the graffiti wall - an oversized piece of paper that appeared upstage of the performance space and became a living document. We thought that the use of the graffiti wall would allow for the cast and the audience to write freely about how they felt in real time. The youth researchers placed key words, thoughts, statements, and themes on the wall as the production was lived and experienced. This added an element of improvisation and helped make the performance relevant and engaging. It also reinforced the note aspect of the Artnote that the youth and audience were collectively creating. The graffiti wall became a document of record - a living document that the audience members could add to after the show. It also served as a record of how the cast felt in the performance as it was unfolding. 


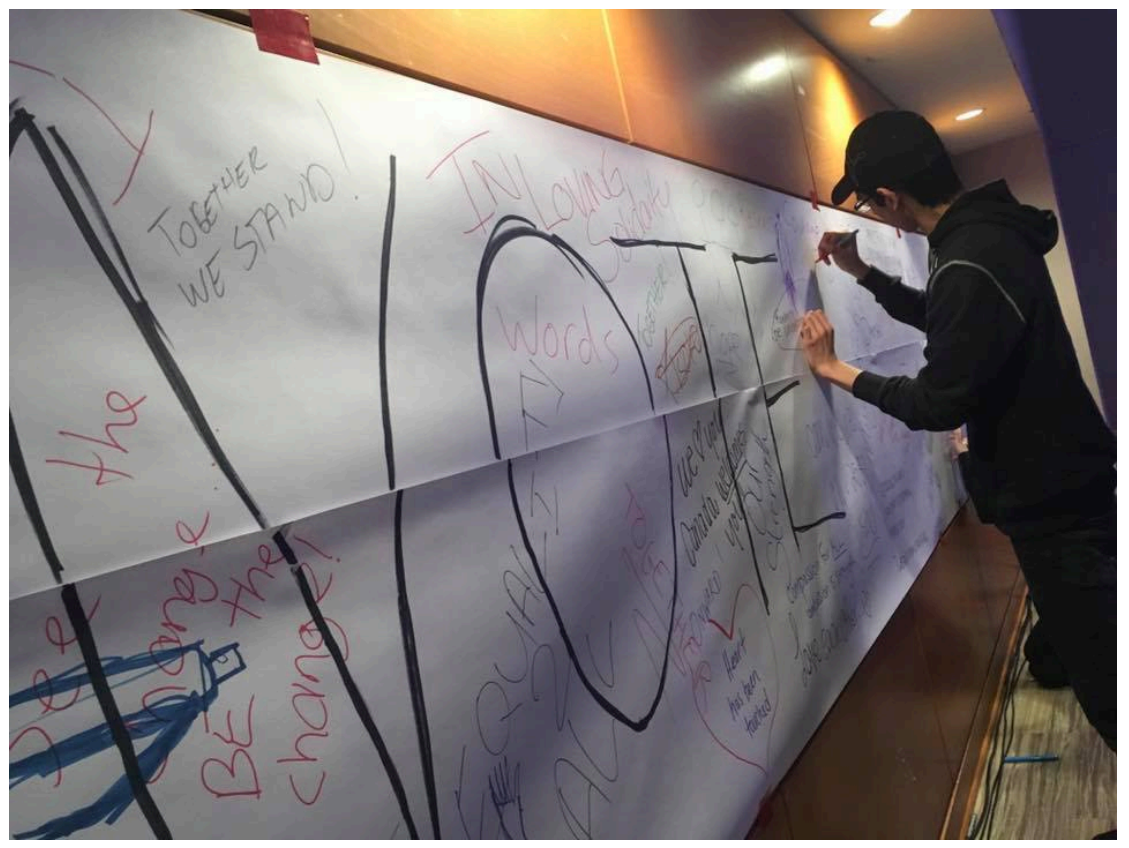

Figure 3. Artnote - The graffiti wall.

We found that the Artnote had a physical, spiritual, and emotional impact on all involved. This impact was the transformative power of understanding which moved us all as Artnote participants to reflect and engage through an embodied/experiential understanding of the research data presented. Dhouha's moving story about leaving her home in the Artnote script illustrated this transformative impact:

I remember we packed up a little suitcase for what was supposed to be a summer vacation in the US with my father. We left our perfect home in Tunisia that summer of 1998. We left everything in place. Our clothes, shoes, furniture, TV's, my books, my artwork on the fridge, and my mother's sewing machine.

I remember the last moment. It is still in my memory. I turned around as we were leaving, and I told myself that this would be the last time that I would see the world that I knew. The world I grew up in. I looked around, I saw the perfectly landscaped and green garden. The one I played in my entire childhood. Where I made my first 
friends and had my first experience of friendship, love, and a sense of community.

As the literature suggest, and we would agree, the arts may offer a lived or experiential approach to the dissemination of research data. Eisner (2001) contended that, "the form of representation one uses has something to do with the form of understanding one secures.... different forms could convey different meanings" (p. 139). We as people naturally learn through doing. However, it could be stated that the human condition is most affected by learning through our experiences (Dewey, 1934, 1938). In speaking about racism and social justice, Bell (2010) postulated that stories, "help us connect individual experiences with systemic analysis, allowing us to unpack in ways that are perhaps more accessible than abstract analysis alone..." and that "stories offer an accessible vehicle for uncovering normative patterns and historical relations that perpetuate racial privilege" (p. 16). The Kosovo youth researchers powerfully expressed these stories based on their research on stage during the Artnote performance by incorporating artifacts and songs of historical, national, and cultural significance in their presentation of research data. 


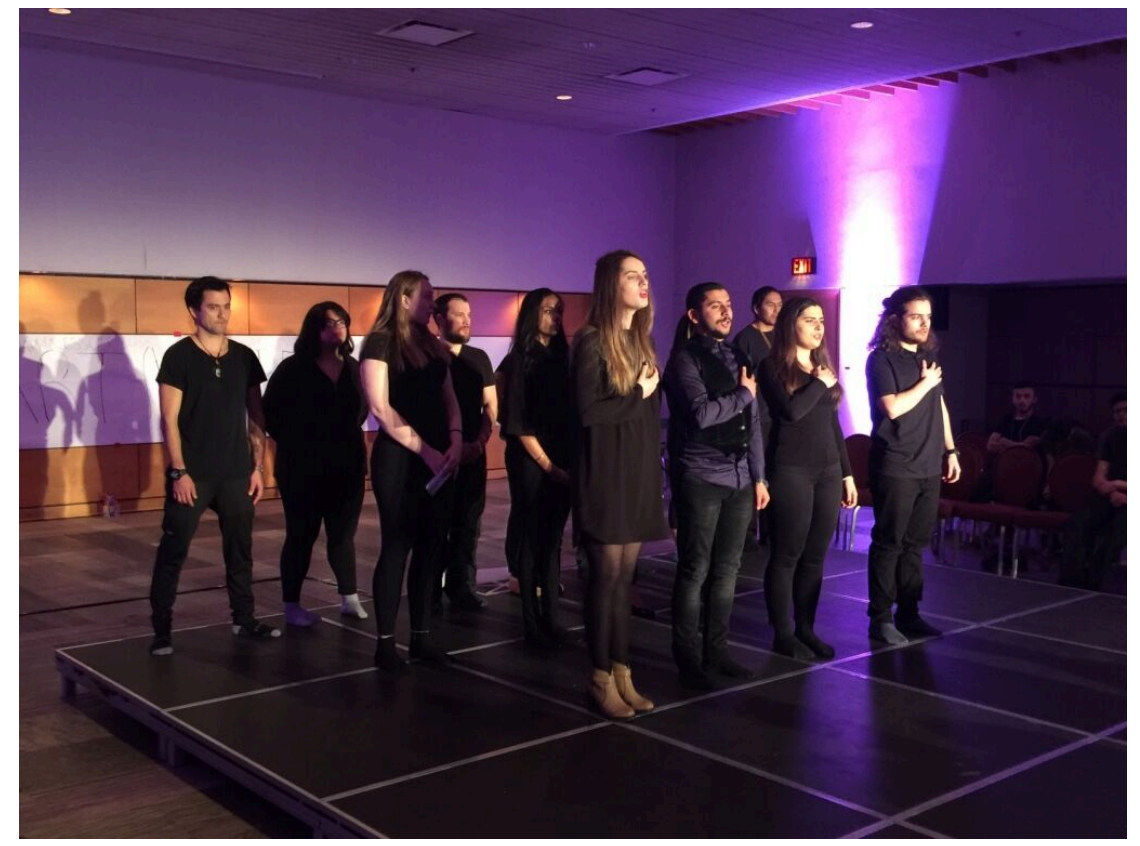

Figure 4. Kosovo youth researchers' Artnote presentation.

\section{Fostering the Voice of Youth Leadership in Social Justice Research and Activism}

Drama comes from the Greek word "to do;" through the Artnote - data as performance, we created a means through which the researchers and audience were living and embodying the research. It is at the core of who we are as humans to be affected by what happens to us personally, and yet, drama may also provide us with an intimate portrait of humanity (Prus, 1996). The Artnote provided a means for youth researchers to express their lived experience, supported by an arts-based experiential approach to connect participants (youth researchers and audience) emotionally with the lived experience of others through the research data presented, including a deeper understanding of perceptions, ideas, and meanings. The authors, working as social justice educators, have witnessed this personal growth during the development of a diverse range of artistic projects and contributions. As such, we have often reflected and examined a range of 
approaches in attempting to determine how we learn. As Nierenberg (2017) suggested, understanding our learning may help us understand the world.

In connecting the various project site performances, the production was organized into pieces, or moving parts. The pieces were connected by looking for the connections that could effectively bridge one performance with the next seamlessly. Working with the Windsor location first really assisted this process. With the Windsor project site, we were able to experiment and try new things to see if they worked - a workshop phase. This workshop phase allowed us to create a framework for the Artnote developed through play and experimentation. Sometimes this workshop phase resulted in a lack of success, but through perseverance it ultimately led to the creation of a performance that conveyed the messages that the youth researchers wished to share. Given their experience in this process, the youth researchers from the Windsor site embraced their role as mentors - mentors who could guide the youth researchers from the other Tikkun project sites through this creative process:

An example of the Windsor host site mentorship happened when developing the opening scene of the Artnote performance. The Tikkun youth researchers from each of the project sites may have been fighting for a different cause in their own communities, but as a collective, they were united in that they were all fighting for what they believed in. Through their mentorship, the Windsor site guided the entire group of youth researchers to collectively decide on a message protesting injustice and activism through a call to action. To illustrate their collective message, the opening scene of the Artnote involved all the youth researchers walking on the stage as protesters. This symbolized their united need for change. It was a moment that brought the audience into their activism - from that moment forward the audience was part of the experience and we broke the fourth wall and allowed the audience to share in the voices of youth. The introduction as a protest rally showcased the voices of the youth!

Picower (2012) stated that, "emerging social justice educators need to develop an orientation toward activism in which they see it as a calling something they can't imagine not doing" (p. 113). A common thread or theme reflected in the stories and impressions shared by the youth researchers 
involved in the Tikkun Youth Project was that they felt that their voices were silenced, that they were not heard by society. However, this did not lead to apathy for social justice; instead, it began the process of coming together - the experience of disseminating their research by sharing their stories resulted in an urgent need to continue to interact with one another for change! During the final Tikkun Youth Symposium wrap-up meeting, youth researchers shared that "we have it within us" to be social innovators and social activists. In particular, the youth researchers indicated that they felt "resource rich" in this regard in terms of personal capital. However, they also felt that they were "resource poor" and that social justice entrepreneurship may be a possible means to fund projects at both the local and global levels. They stressed the importance of continuing to learn from each other, networking, and sharing techniques and strategies - building and sharing the skills of protest and advocacy... moving forward, one step at a time!

\section{Conclusion}

In conclusion, a key element in the creation and performance of the Artnote involved a discussion of "long-form artistic works" versus "short-term utopias" within the artistic landscape. Based on the Broken City Lab's (2013) workshop, Homework II: Long Forms/Short Utopias, which explored this aspect or element of the artistic process, the importance of focusing on the longevity of the art form to thrive as a medium for social change was critical. The concept of focusing on a future-oriented longevity of protest and advocacy clearly resounded in the sentiments expressed by the youth researchers. In other words, the youth researchers recognized that social change for justice is a lengthy endeavour of continual activism, requiring a personal as well as a collective commitment to advocate for those marginalized in society. They recognized that although their work in each community had a specific context, there was nonetheless a thread of connectivity in their activism and advocacy, and that building capacity as a Youth Network may in fact foster healing and reconciliation. What worked for one site might address an issue in another community. Just as the wind creates ripples upon the water in a pond, it is our hope that the experiences that the youth researchers shared during the Tikkun Youth Project - and 
the Artnote performance itself - will support their continued courage and drive to be the waves of change as social justice innovators and leaders, both locally as well as globally.

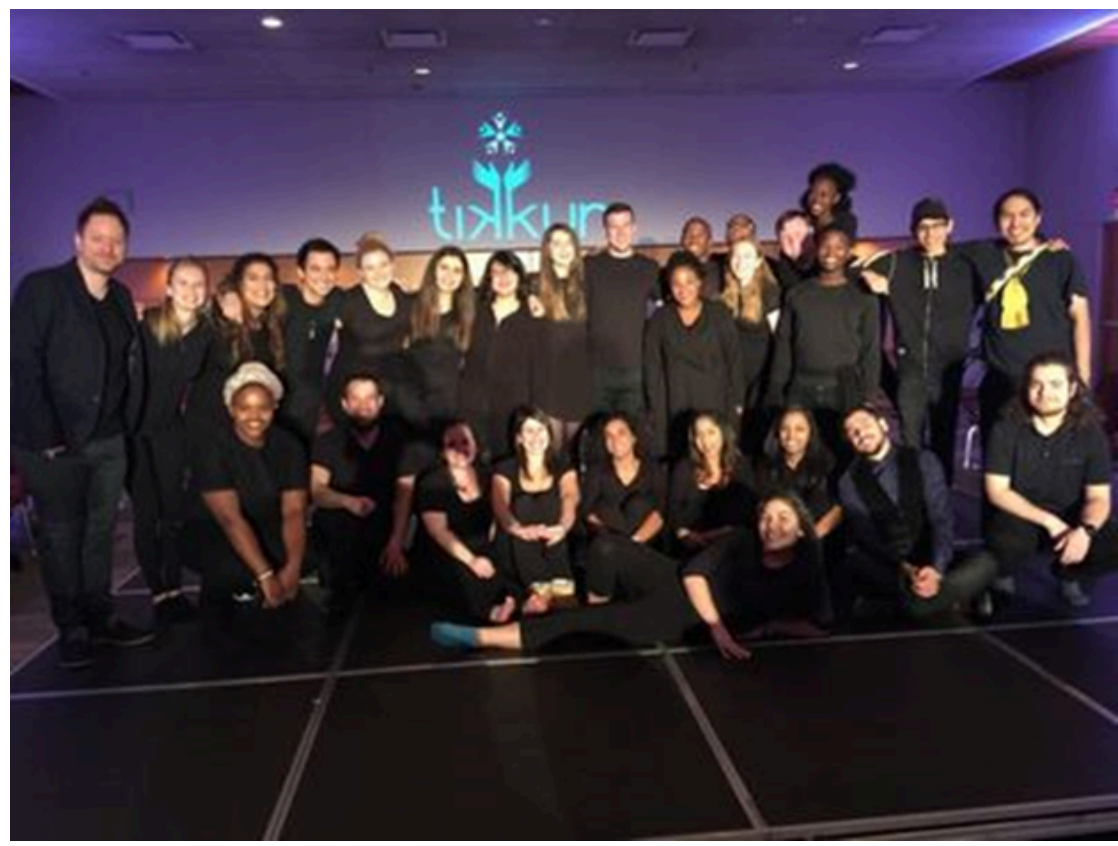

Figure 5. Tikkun youth researchers - Symposium Artnote performance.

\section{References}

Bagley, C. (2008). Educational ethnography as performance art: Towards a sensuous feeling and knowing. Qualitative Research, 8(1), 53-7. Retrieved from doi:10.1177/1468794107085296

Bell, L. A. (2010). Storytelling for social justice: Connecting narrative and the arts in antiracist teaching. New York: Routledge Taylor \& Francis Group.

Broken City Lab. (2013). Homework II: Long Forms/Short Utopias. Steve Lambert. Retrieved from https://visitsteve.com/news/talks/keynote-athomework-ii-long-forms-short-utopias-conferencenovember-8-10-2013/ 
Dewey, J. (1934). Art as experience. New York: Minton, Balch \& Company.

Dewey, J. (1938). Experience $\mathcal{E}$ education. New York: Macmillan Publishing Company.

Eisner, E. (2001). Concerns and aspirations for qualitative research in the new millennium. Qualitative Research, 1(2), 135-45. Retrieved from doi:10.1177/ 146879410100100202

Freire, P. (1985). The politics of education: Culture power and liberation. Westport, CT: Bergin \& Garvey.

Nierenberg, C. (2017, June 2). Knowing yourself: How to improve your understanding of others. Live Science. Retrieved from https://www.livescience.com/59349-knowing-yourself-helps-yourunderstand-others.html

Picower, B. (2012). Practice what you teach: Social justice education in the classroom and the streets. New York: Routledge Taylor \& Francis Group.

Prus, R. (1996). Symbolic interaction and ethnographic research: Intersubjectivity and the study of human lived experience. New York: New York Press.

Tuckman, B. W., \& Jensen, M. A. (1977). Stages of small-group development revisited. Group \& Organization Studies, 2(4), 419-427. Retrieved from doi:10.1177/105960117700200404

1 See Arts Collective Theatre for more information about Chris and his social justice work: http://actwindsor.com/. 